\title{
Making OpenID mobile and privacy-friendly
}

Faysal Boukayoua and Karel Dewitte and Vincent Naessens

\begin{abstract}
OpenID is a widely used single sign-on standard that allows users to access different services using the same authentication. However, its usage poses a number of issues regarding privacy and security. This paper evaluates the OpenID standard and introduces three mobile strategies, two of which are validated using a prototype implementation. Significant privacy and trust improvements are attained through the use of an identity management architecture that leverages the properties of a tamperproof module. Furthermore, our approach makes OpenID more suitable for omnipresent mobile use. We remain interoperable with the OpenID standard and no modifications to the mobile platform are required.
\end{abstract}

Acknowledgements This work was made possible through funding from the MobCom and SecureApps projects, granted by the Flemish agency for Innovation by Science and Technology (IWT).

\section{Introduction}

It goes without saying that Web services have become commonplaces of everyday life. Their mobile use has soared during the past years. Authentication remains an important concern throughout this evolution. A well-established and popular standard is OpenID. Many online parties provide their users with proprietary authenti-

Faysal Boukayoua

KU Leuven, Department of Computer Science, Technology Campus Ghent, Gebroeders De Smetstraat 1, 9000 Ghent, Belgium, e-mail: faysal.boukayoua@cs.kuleuven.be

Karel Dewitte

KU Leuven, Department of Computer Science, Technology Campus Ghent, Gebroeders De Smetstraat 1, 9000 Ghent, Belgium, e-mail: karel.dewitte@hotmail.com

Vincent Naessens

KU Leuven, Department of Computer Science, Technology Campus Ghent, Gebroeders De Smetstraat 1, 9000 Ghent, Belgium, e-mail: vincent.naessens@cs.kuleuven.be 
cation accounts that are OpenID-enabled. This frees users from having to maintain different accounts and they only have to sign in once to access services that support OpenID.

Boukayoua et al [2] outline a number of complementary weaknesses and strengths in network-based and claim-based identity management systems. In a networkbased system, storage of user attributes is centralised at the identity provider, making it a high-value attack target. In many such systems, this actor is also directly involved in the authentication, increasing its capabilities to monitor, link and profile user transactions. On the other hand, network-based systems are typically more standardised than claim-based ones and they also require less modification to workstations. The authors present a hybrid architecture to leverage the strengths of both systems and to mitigate their weaknesses. It is validated in this work with OpenID as the network-based part and the architecture by Vossaert et al [16] as the claim-based part.

Contribution: This paper addresses multiple privacy and security issues in OpenID. We present three strategies to make it more secure and privacy-friendly. The three are compared in terms of privacy, trust, security and interoperability properties. Two of the three strategies are validated with a prototype implementation. Additionally, OpenID is made more suitable for today's authentication needs, as all three approaches incorporate commodity mobile devices.

The remainder of this paper is structured as follows. Section 2 provides further background and gives an overview of related work. The integration strategies are put forward in section 3 . The prototype implementations are described in section 4. while section 5 presents an evaluation and comparison of the three approaches. Finally, conclusions are drawn in section 6

\section{Background and related work}

OpenID is a network-based single sign-on protocol. It allows the user to prove ownership of an identifier, typically a URL. Version 2 of the standard was made public in 2007 [9].

To start authenticating, the user provides his identifier to the relying party (1). Using this identifier, the relying party carries out a discovery, the result of which is the identity provider's URL (2). Next, a session is established between the relying party and the identity provider (3). The association that OpenID provides here, is insufficient. As the standard does not mandate the use of TLS1 it is left vulnerable to man-in-the-middle attacks [7]. In a next step, the user is redirected to the identity provider (4), where he is authenticated (5). In case of success, he is supplied with an assertion from the identity provider and redirected back to the relying party (6). In the assertion, the identity provider provisions any required attributes and endorses

\footnotetext{
${ }^{1}$ Transport Layer Security: the successor of SSL.
} 
the user's ownership of the given identifier. If it is authorised by the relying party (7), the user can now access the protected resource (8).

Being a standard, OpenID is well-established and widely deployed. However, its use incurs several security issues. Many OpenID providers resort to passwords, which are often reused and chosen weak [17, 15]. An exception is myOpenID ${ }^{2}$, which offers certificate-based authentication. Furthermore, OpenID is very prone to phishing. Instead of redirecting the user to his identity provider, a malicious relying party can easily present a self-constructed page, where login info is captured [4, 7]. In addition, since an OpenID account is used for single sign-on, a permanently authenticated session with the identity provider is not uncommon. If the latter does not implement proper user authorisation, cross-site request forgery (CSRF) allows an attacker ${ }^{3}$ to authenticate to other relying parties on behalf of the user [4, 1]. Once logged in, further exploitation could allow the adversary to perform privileged actions. Additionally, malware on the workstation can intercept the user name and password. What's more, multiple security-critical responsibilities are fulfilled by the identity provider: authentication, attribute storage, as well as provisioning. This makes it a high-value attack target [2].

OpenID performs poorly in terms of trust properties [7]. The identity provider is not required to be a trusted party, as opposed to, f.i., Shibboleth [14]. It could as well be under the user's control. Proving ownership of an identifier is by no means equivalent to making assertions about a user's identity! In the first years of OpenID, the user could provide any identifier, a practice still in use. Later, many relying parties restricted this to a handful of trusted identity providers (Google, Facebook,...).

OpenID exhibits a number of privacy issues. Typically, a globally unique, DNSbased identifier is used, making user transactions linkable by relying parties. In addition, the identity provider's intervention is required for each authentication, giving it pervasive capabilities for monitoring, linking and profiling [5, 77]. Since the identity provider and the relying party are in direct contact, this allows them to collude against the user. Furthermore, many implementations are not equipped to show user feedback and to ask for consent. Others, like Google, display a feedback and consent prompt only upon first sign-on to a relying party. To the best of our knowledge, there is not yet an OpenID version that offers selective attribute disclosure, similar to what Shibboleth's uApprove [10] does.

This work considers OpenID in combination with the identity management architecture (onward referred to as the IdM architecture) by Vossaert et al [16]. It exploits the properties of a tamperproof module to enforce trust. A strict separation between claim provider ${ }^{4}$ and relying parties is maintained, as their communication is mediated through the tamperproof module. The middleware displays feedback about the ongoing transaction and allows the user to consent, which includes entering his PIN. Identifiable, pseudonymous as well as (accountable) anonymous transactions are supported. Standalone mobile use of this architecture was previously validated

\footnotetext{
2 https://www.myopenid.com

${ }^{3}$ This can be a malicious website or an attacker infecting a trusted website.

${ }^{4}$ To avoid confusion, claim-based identity providers are referred to as claim providers.
} 
by Boukayoua et al [3]. However, real-life use requires an approach that is more interoperable with existing infrastructure.

Related research is presented by Dodson et al. [8], proposing a phone-based, asymmetric challenge-response protocol for user authentication to the OpenID provider. The approach by Feld et al [11] is similar, which combines the German eID with OpenID. Both approaches offer protection against phishing and replace user names and passwords by strong authentication. However, they do not propose a trust infrastructure to endorse users' identity information. In addition, the OpenID provider still stores user information, thus remaining a high-value attack target. Leicher et al. [13] integrate the infrastructure of a mobile phone operator into OpenID. Added to the improvements of the two previous works, this approach introduces trust in OpenID by relying on the operator's infrastructure and on the tamperproofness of a SIM card. They also extend OpenID with support for pseudonymity and anonymity. However, the focus mainly lies on how to combine OpenID with the infrastructure of a mobile network operator.

\section{Approach}

To resolve the OpenID issues that are listed in section 2, three different approaches are introduced. First, we list the requirements we aim to satisfy.

\subsection{Requirements}

The security requirements are as follows:

$S_{1}$ Passwords are substituted for stronger credentials.

$S_{2}$ Protection against phishing is provided.

$S_{3}$ Authentication-related CSRF attacks are prevented.

$S_{4}$ The attack value and exposure of the identity provider is reduced.

$S_{5}$ Data authentication and confidentiality is ensured between the communicating parties.

To address the privacy issues, we require the following:

$P_{1}$ The identity provider's capabilities for transaction monitoring, linking and profiling, are reduced.

$P_{2}$ Collusion between identity providers and relying parties is obstructed.

$P_{3}$ The identity provider cannot impersonate the user.

$P_{4}$ The user can selectively disclose or withhold his attributes.

As for trust, we impose that:

$T_{1}$ relying parties can corroborate provisioned user attributes. 
$T_{2}$ the required trust in the workstation is reduced, as this can be a publicly used computer.

The remaining requirements pertain to interoperability and usability:

$R_{1}$ We remain compliant with the OpenID standard.

$R_{2}$ No modifications are applied to the relying party's infrastructure.

$R_{3}$ The relying party is agnostic to the claim-based credential technology in use.

$R_{4}$ The solution is portable across workstations.

$R_{5}$ Feedback about the ongoing transaction is presented to the user and his consent is enforced.

\subsection{Improvement strategies}

Each approach shifts a gradually increasing part of the identity provider's responsibilities towards the user's smartphone and/or to the tamperproof module.

\subsubsection{Strategy 1: authentication via the mobile device, attribute storage and provisioning by the OpenID identity provider.}

In this setup, two major changes are applied to a password-based OpenID implementation. First, a mobile device with a tamperproof module is introduced. The latter contains the mobile part of the IdM architecture. It is addressed by the phone through a middleware layer. Second, password-based authentication is substituted for the one used in the IdM architecture, Elliptic Curve Diffie-Hellman. The user is authenticated out of band and his consent is first needed to proceed. Note that the identity provider still stores and provisions user attributes.

As a result, the - typically password-based - authentication in the standard protocol, takes place as follows. Using a short-range communication protocol, a session challenge is advertised to the authentication app on the user's mobile device. The app displays information about the identity provide $\mathrm{i}^{5}$ and asks for the user's permission to proceed. Upon confirmation, the challenge is sent to the tamperproof module. Prior to setting up a secure channel with the identity provider, it prompts the user to enter his PIN. The authentication response is sent over this channel. After this authentication response is verified, the standard OpenID protocol flow is resumed.

As mentioned in section 2, OpenID is vulnerable to phishing attacks by the relying party. To mitigate this, our authentication app expects the user to preconfigure a list of trusted identity providers.

\footnotetext{
${ }^{5}$ From the viewpoint of the IdM architecture, the OpenID identity provider is essentially a relying party, in this case requiring authentication without attribute provisioning.
} 


\subsubsection{Strategy 2: authentication and attribute provisioning by a tamperproof module on the device}

The concerns and steps of this approach are largely similar to 3.2 .1 . Here however, attribute storage and provisioning are also moved to the IdM architecture. Therefore, the remaining responsibility of the identity provider is to convert claims to OpenID assertions that are understood by the relying party. The user still authenticates to the identity provider. However, he must now also agree to disclose attributes to the relying party and, if yes, indicate which ones to release. These are transferred over the secure channel that is set up upon authentication [16]. Consequently, the attributes the identity provider requires, are part of the challenge that is advertised to the mobile device. They are displayed as feedback to the user, alongside the relying party to which info is about to be disclosed. Note that the identity provider does not create the OpenID assertion using information from its own data storage. Instead, it uses the attributes that are provisioned by the tamperproof module.

\subsubsection{Strategy 3: the mobile device running an entire OpenID identity provider}

In this setup, the identity provider's functionality is entirely moved to the user's mobile and tamperproof module ( $\mathrm{mldP}$ ). It consists of two components: a lightweight Web server and an authentication app. This app addresses the tamperproof module for security- and trust-sensitive operations. The resulting protocol is as follows. The user requests a protected resource, upon which the relying party asks for and receives his OpenID identifier. The relying party then obtains the mldP's address through the discovery step. Subsequently, the mIdP and the relying party set up a shared session over a secure channel. The browser is then redirected to the mldP and the user is prompted on the mobile device to review and agree to the attributes to provision and the relying party to disclose them to. Following his user's consent, the challenge and the list of required attributes are sent to the tamperproof module. The latter prompts the user to enter his PIN. If successful, an authentication and assertion response is generated. The mldP then redirects the user's browser to the relying party and sends along the generated response. If it is correctly verified, the protected resource is made available to the user.

Note that this approach authenticates the tamperproof module in step 5: the IdM architecture's common key [16] is used to establish a TLS session between the relying party and the mldP. Also note that no short-range communication is required to transfer the authentication challenge from the browser to the mobile device. 


\subsection{Trust relations and enforcement}

As section 2 points out, OpenID falls short regarding trust establishment. On the other hand, many corporations, governments and telcos already have trust infrastructures in place. Reusing them is both cost-effective and an added value to users and relying parties.

In strategy 1 , the identity provider needs to establish trust in the claim providers and the issuer of the tamperproof module. If all three fall under the same organisation, they are likely part of the same PKI If not, the identity provider must implement a black- or whitelisting mechanism. Trust is enforced by the tamperproof module and the secure channel that it maintains towards the identity provider [16]. The relying party trusts the user authentication by the identity provider. This trust is enforced by (1) TLS for direct communication between the identity provider and the relying party (2) $\mathrm{MAC}^{7}$ authentication for communication that is mediated by the browser. The MAC key is part of the pre-established session between the relying party and the identity provider.

Similar considerations apply to the second strategy. In addition, since the identity provider now acts as an attribute mediator between the tamperproof module and the relying party, the latter needs to express which claim providers it trusts. This trust preference can be indicated in the OpenID authentication request. Alternatively the relying party can accept all claim providers that are also trusted by the identity provider. The first approach allows fine-grained control, while the second is realisable without cooperation from the identity provider.

In the third strategy, the relying party needs to establish trust in each mobile identity provider, i.e. in the issuers of its tamperproof module and in the credentials that are stored on it. Additionally, the IdM architecture can also restrict the disclosed attributes per relying party [16].

\section{Proof of concept}

This section describes the proof of concept, in which strategies 1 and 3 are validated. Only the infrastructure of the user and the identity provider are addressed, as the relying party is not modified (see section 3.1).

\subsection{Strategy 1}

The identity provider runs in a lightweight Jetty Servlet container, both suitable for embedded devices and large-scale computing applications. The OpenID imple-

\footnotetext{
${ }^{6}$ Public Key Infrastructure.

${ }^{7}$ Message Authentication Code.
} 
mentation is OpenID4Java. Memcached, an in-memory key-value store, maintains session statuses between the browser and the identity provider. A session is marked as authenticated upon successful sign-on.

QR codes are used for short-range communication between the browser and the mobile device. These are two-dimensional barcodes that can contain up to $3 \mathrm{kB}$ of data. They offer multiple advantages. No extra hardware or configuration is required, as opposed to Bluetooth. The requirement of a computer screen and a smartphone camera are essentially no limitation. As authentication takes place out of band, a mechanism is required to resume the browser's workflow. A status checking page is created for this purpose. The QR authentication page contacts it, using an asynchronous long-polling reques 8 the artificially long timeout allows the smartphone authentication to complete before returning an HTTP status - 200 in case of success. To make the session known to the phone, the QR code contains its identifier. This is, however, an alternate id, to obstruct shoulder surfing. The mapping between both ids is only known to the identity provider.

The mobile device is an Acer Liquid Glow with Android 4. Smartphones are ideal for authentication, considering their widespread presence and personal nature. As a tamperproof module, we use a Giesecke \& Devrient Mobile Security Card SE 1.0, running JavaCard 2.2.2. An authentication app reads the QR code using the smartphone camera, extracts the identity provider's information and displays it to the user for reviewing. The tamperproof module generates a response and sends it over a secure channel to the identity provider.

\subsection{Strategy 3}

In this strategy, the identity provider entirely resides on the tamperproof module and on the mobile device. Feedback and consent, communication with the tamperproof module and the authentication and attribute provisioning servlets are all confined to one Android application. This is preferred over a multi-app approach, as it poses less performance overhead. More importantly, it avoids inter-process communication and its related security concerns [6]. Additionally, it is also more portable to platforms with limited capabilities for inter-process communication. The same phone and tamperproof module as in strategy 1 are used. Note that no short-range communication is required.

TLS sessions use the card-specific common key [16] to authenticate the mobile identity provider to the relying party. The Mobile Security Card provides private key operations through a PKCS\#11 interface [12]. Unfortunately, the card's processing power is not yet sufficient to handle the exchange of all OpenID messages with the relying party over an end-to-end channel. Therefore, message generation and parsing takes place on the phone rather than on the tamperproof module. We expect

${ }^{8}$ Repeated polling incurs greater networking overhead and HTTP WebSockets are not supported by older browsers. 
this hurdle to be resolved soon, as new and more powerful tamperproof modules find their way to the market.

\section{Evaluation}

\subsection{Requirements evaluation}

This section evaluates how well the requirements from section 3.1 are satisfied. An overview is provided in table 1 All three strategies substitute passwords for strong authentication (S1). Furthermore, as strategy 1 and 2 expect the user to preconfigure a trusted list of identity providers, phishing on the workstation is obstructed (S2). As for CSRF attacks, strategy 3 entails no session between the browser and the mobile identity provider. The user's authorisation is requested for every transaction, thus preventing authentication-related CSRF attacks (S3). While attributes are still stored by the identity provider in strategy 1 , they are decentralised in strategies 2 and 3. This reduces the exposure and attack value of the identity provider (S4). As for data authentication and confidentiality, either TLS or the IdM architecture's end-to-end secure channel [16] is used between every two communicating parties (S5). Strategies 1 and 2 do not protect against monitoring, linking and profiling by the identity provider, which is directly involved in every transaction. Strategy 3 resolves this by placing the entire identity provider on the user's mobile device and tamperproof module (P1-P3). Furthermore, the user is provided with the means to review and consent to each transaction (P4). Section 3.3 explains how to reuse existing infrastructures to make OpenID trust-enabled (T1). Additionally, none of the three approaches involves entering credentials on the workstation (T2). This is attained at the expense of requiring more trust in the mobile device. This is, however, a reasonable choice, considering the more elaborate security model of today's mobile platforms and the use of a tamperproof module. Also, the user is likely to trust his mobile device more than a public computer for authentication.

As for the other requirements, none of the approaches modify the OpenID standard (R1). Furthermore, the relying party's infrastructure does not need to change (R2), with the exception of optionally maintaining black- or whitelists of claim providers and/or tamperproof module issuers (see section 3.3. Furthermore, the relying party is not exposed to details of any claim-based credential technology (R3). The shift towards mobile devices, makes the three strategies portable across workstations (R4). Lastly, all three also use the mobile device to display feedback about the ongoing authentication (R5). 


\begin{tabular}{|l|l|l|l|l|}
\hline & $\begin{array}{l}\text { Password-based } \\
\text { OpenID }\end{array}$ & Strategy 1 & Strategy 2 & Strategy 3 \\
\hline $\begin{array}{l}\text { Strong authentication } \\
\text { instead of passwords }\end{array}$ & IdP-dependent & Yes & Yes & Yes \\
\hline Phishing protection & No & Yes & Yes & Yes \\
\hline $\begin{array}{l}\text { Protection against authN- } \\
\text { based CSRF attacks }\end{array}$ & IdP-dependent & IdP-dependent & IdP-dependent & Yes \\
\hline $\begin{array}{l}\text { Reduced attack value of IdP } \\
\text { Data authN and confiden- } \\
\text { tiality between parties }\end{array}$ & \begin{tabular}{ll|l} 
IdP- and RP- \\
dependent
\end{tabular} & RP-dependent & RP-dependent & RP-dependent \\
\hline $\begin{array}{l}\text { Reduced linking monitor- } \\
\text { ing, profiling by IdP }\end{array}$ & No No & No & Yes \\
\hline $\begin{array}{l}\text { Prevent collusion between } \\
\text { IdP and RP }\end{array}$ & No (IdP and RP communicate directly) & Yes \\
\hline $\begin{array}{l}\text { User impersonation by IdP } \\
\text { prevented }\end{array}$ & No & No & No & Yes \\
\hline Selective attribute disclosure & IdP-dependent & IdP-dependent & Yes & Yes \\
\hline Reduced trust in workstation & No & Yes & Yes & Yes \\
\hline
\end{tabular}

Table 1 Comparison of how well each strategy satisfies the privacy, security and trust requirements $(\mathrm{RP}=$ relying party, $\mathrm{IdP}=$ identity provider $)$

\subsection{Network anonymity}

Mobile devices are usually tied to an individual user. Therefore, strategy 3 may lead to increased network anonymity concerns: a mobile identity provider with a publicly unique IP address, allows the relying party to link transactions. Approaches to mitigate this, must ensure that the mobile identity provider remains reachable as a Web service. The simplest approach is for the user to rely on a frequently changing IP address. However, this is rarely under his control. Alternatively, a reverse proxy service can (1) be shared by multiple devices (2) generate random, changing subdomains or URL segments that correspond to each mobile identity provider. However, this requires trust in the party that operates this proxy. Another possibility is for the mobile identity provider to register as a hidden service on anonymity networks like Tor or $\mathrm{I}^{2} \mathrm{P}$. Typical use, however, involves both the relying party and the mobile identity provider being connected to such a network. In the case of Tor, this can be mitigated by using Tor2web. This is a widely hosted WWW-to-Tor gateway software, allowing the exposure of anonymised websites to clients that are not running Tor.

\subsection{Discussion}

The interoperability and usability requirements are fulfilled by all three strategies. Furthermore, a tradeoff can be observed in terms of infrastructural prerequisites and privacy and security gains. The merits of strategy 1 are secure authentication and de- 
creased trust in the workstation. In return, a mobile device with the IdM architecture is needed. The privacy benefits are limited. Strategy 2 moves attribute provisioning to the mobile device. This reduces the attack value of the OpenID provider. It also also allows the user to selectively disclose his attributes. However, this strategy entails far-reaching modifications to the identity provider, in which significant trust is still required. Strategy 3 offers the best privacy properties. It does away completely with the OpenID provider as a third party. As a result, the user has full control over his transactions and over the attributes he discloses. Here however, the mobile device must be reachable as an online server. Additionally, network anonymity becomes an important concern, as mobile devices are typically personal.

\section{Conclusion}

This paper has presented three hybrid strategies to address multiple security and privacy issues in OpenID. Properties like trust and interoperability are also taken into account. Through a comparative evaluation, we have shown significantly better performance -each strategy to its own extent- than existing OpenID implementations. The use of commodity mobile devices makes OpenID more suitable for today's authentication needs. No modifications to OpenID nor the mobile platform are needed. Two of the three strategies are validated by a prototype.

\section{References}

1. Adam Barth, Collin Jackson, and John C. Mitchell. Robust defenses for cross-site request forgery. In Proceedings of the 15th ACM conference on Computer and communications security, CCS '08, pages 75-88, New York, NY, USA, 2008. ACM.

2. Faysal Boukayoua, Jan Vossaert, Bart Decker, and Vincent Naessens. Claim-based versus network-based identity management: A hybrid approach. In Security and Privacy in Mobile Information and Communication Systems, volume 107 of Lecture Notes of the Institute for Computer Sciences, Social Informatics and Telecommunications Engineering, pages 38-50. Springer Berlin Heidelberg, 2012.

3. Faysal Boukayoua, Jan Vossaert, Bart Decker, and Vincent Naessens. Using a smartphone to access personalized web services on a workstation. In Privacy and Identity Management for Life, volume 375 of IFIP Advances in Information and Communication Technology, pages 144-156. Springer Berlin Heidelberg, 2012.

4. Stefan A. Brands. The problem(s) with openid. http://untrusted.ca/cache/ openid.html, August 2007.

5. David W. Chadwick. Federated identity management. In Alessandro Aldini, Gilles Barthe, and Roberto Gorrieri, editors, Foundations of Security Analysis and Design V, chapter Federated Identity Management, pages 96-120. Springer-Verlag, Berlin, Heidelberg, 2009.

6. Erika Chin, Adrienne Porter Felt, Kate Greenwood, and David Wagner. Analyzing interapplication communication in android. In Proceedings of the 9th International Conference on Mobile Systems, Applications, and Services, MobiSys '11, pages 239-252, New York, NY, USA, 2011. ACM. 
7. Bart Delft and Martijn Oostdijk. A security analysis of openid. In Elisabeth Leeuw, Simone Fischer-Hübner, and Lothar Fritsch, editors, Policies and Research in Identity Management, volume 343 of IFIP Advances in Information and Communication Technology, pages 73-84. Springer Berlin Heidelberg, 2010.

8. B. Dodson, D. Sengupta, D. Boneh, and M. S Lam. Secure, consumer-friendly web authentication and payments with a phone. In Conference on Mobile Computing, Applications, and Services (MobiCASE'10), Santa Clara, CA, USA, 2010.

9. Fitzpatrick et al. OpenID authentication 2.0 - final, December 2007.

10. Orawiwattanakul et al. User-controlled privacy protection with attribute-filter mechanism for a federated sso environment using shibboleth. In Proceedings of 3PGCIC 2010, 3PGCIC '10, pages 243-249, Washington, DC, USA, 2010. IEEE Computer Society.

11. Sebastian Feld and Norbert Pohlmann. Security analysis of openid, followed by a reference implementation of an npa-based openid provider. In ISSE 2010 Securing Electronic Business Processes, pages 13-25. Vieweg+Teubner, 2011.

12. RSA Laboratories. Pkcs \#11 v2.30: Cryptographic token interface standard. http://www.emc.com/emc-plus/rsa-labs/standards-initiatives/ pkcs-11-cryptographic-token-interface-standard.htm April 2009.

13. Andreas Leicher, Andreas U. Schmidt, and Yogendra Shah. Smart OpenID: a smartcardbased OpenID protocol. In Proceedings of the 27th IFIP International Information Security and Privacy Conference, Heraklion, Crete, Greece, June 2012. Springer.

14. R. L. Morgan, Scott Cantor, Steven Carmody, Walter Hoehn, and Ken Klingenstein. Federated security : The shibboleth approach. EDUCAUSE Quarterly, 27(4), 2004.

15. Denise Ranghetti Pilar, Antonio Jaeger, Carlos F. A. Gomes, and Lilian Milnitsky Stein. Passwords usage and human memory limitations: A survey across age and educational background. PLoS ONE, 7(12):e51067, 122012.

16. Jan Vossaert, Jorn Lapon, Bart De Decker, and Vincent Naessens. User-centric identity management using trusted modules. In Proceedings of the 7th European conference on Public key infrastructures, services and applications, EuroPKI'10, pages 155-170, Berlin, Heidelberg, 2011. Springer-Verlag.

17. Jeff Yan, Alan Blackwell, Ross Anderson, and Alasdair Grant. Password memorability and security: Empirical results. IEEE Security and Privacy, 2(5):25-31, September 2004. 